Check for updates

Cite this: RSC Adv., 2017, 7, 53334

Received 2nd August 2017

Accepted 6th November 2017

DOI: $10.1039 / c 7 r a 08514 a$

rsc.li/rsc-advances

\section{Non-additive impacts of covalent cross-linking on the viscoelastic nanomechanics of ionic polyelectrolyte complexes}

\author{
Biao Han, ${ }^{a}$ Tianzhu Ma, ${ }^{a}$ John H. Vergara, ${ }^{b}$ Giuseppe R. Palmese, ${ }^{b}$ Jie Yin, ${ }^{c}$ \\ Daeyeon Lee (iD ${ }^{d}$ and Lin Han (iD *a
}

\begin{abstract}
This study elucidates the influences of adding covalent cross-linking on the nanomechanical viscoelasticity of ionically cross-linked polyelectrolyte networks. Using layer-by-layer (LbL) assembled PAH/PAA networks, we report how adding covalent amide cross-links changes the degree of swelling, indentation modulus, and force relaxation behaviors with varying solution conditions such as $\mathrm{pH}$ and ionic strength, which modulate the ionic cross-link density and fixed charge density. The addition of covalent cross-linking increases both the modulus and degree of elasticity through providing permanent anchorage to the ionically linked networks. Such addition also strongly increases the relaxation times at net neutral, less swollen states. These results together underscore the synergistic interplay between the covalent and ionic cross-links in the viscoelastic nanomechanics of polyelectrolyte networks, potentially enabling the use of these stimulus-responsive materials in mechano-sensitive biomedical and engineering applications.
\end{abstract}

\section{Introduction}

Owing to their responsiveness to stimuli, ease of manufacturing and nano-to-microscale dimensions, layer-by-layer (LbL) assembled polyelectrolyte films have shown potential in a wide range of biomedical applications, such as cell substrates, ${ }^{\mathbf{1 - 5}}$ biomaterial coatings, ${ }^{6,7}$ optical coatings, ${ }^{8}$ anti-fouling coatings, ${ }^{\mathbf{9}, 10}$ drug delivery carriers, ${ }^{11}$ and tissue engineering. ${ }^{12,13}$ Upon assembly, polyelectrolyte chains form networks held by physical cross-links, such as ionic bonding, ${ }^{\mathbf{1 4}}$ hydrogen bonding ${ }^{15}$ and hydrophobic interactions. ${ }^{16}$ The highly dynamic, environment-sensitive nature of these physical cross-links endows the films with highly tunable optical, mechanical and electrical properties. ${ }^{\mathbf{1 7}}$ However, physical cross-links can easily break under mechanical stress or upon environmental changes; the as-prepared, physically linked films thus do not provide structural stability in comparison to covalently linked films. Therefore, introducing additional covalent cross-links has become a common practice to increase the film stability. ${ }^{18}$ As a result of the additional cross-linking, the LbL films become microscale polyelectrolyte networks, held by a mixture

${ }^{a}$ School of Biomedical Engineering, Science, and Health Systems, Drexel University, Philadelphia, PA 19104, USA. E-mail: lh535@drexel.edu; Fax: +1 215895 4983; Tel: +12155713821

${ }^{b}$ Department of Chemical and Biological Engineering, Drexel University, Philadelphia, PA 19104, USA

'Department of Mechanical Engineering, Temple University, Philadelphia, PA 19122, USA

${ }^{d}$ Department of Chemical and Biomolecular Engineering, University of Pennsylvania, Philadelphia, PA 19104, USA of permanent, covalent cross-links and dynamic, stimulusresponsive physical cross-links.

This hybrid cross-linking nature indicates that the mechanical behaviors of such films are likely different from those held by single cross-link type, either covalent or physical. Indeed, at the macroscale, hydrogels with two cross-link types have shown unique, advantageous properties, such as increased toughness, ${ }^{19,20}$ improved resistance in enzymatic degradation, ${ }^{21}$ and tunable surface chemistry. ${ }^{22}$ Therefore, hybrid cross-linking serves as an important variation of the LbL technique to meet the requirements for various applications. To date, for these films, at the microscale, it is well-known that adding covalent cross-links significantly increases the elastic modulus. ${ }^{23-26}$ However, given the deformation of polymer chains is timedependent, a salient feature of polymer mechanics is viscoelasticity. It is unclear how this mixture of cross-link types can yield viscoelastic behaviors distinct from those held solely by physical or covalent cross-links. Since many applications rely on the mechanical properties of these films, ${ }^{27-29}$ understanding viscoelasticity at the microscale will lay the ground for the design of various mechano-sensitive biomedical and engineering applications using LbL films.

The objective of this study was to determine the impact of covalent cross-linking on the viscoelastic mechanical properties of ionic LbL polyelectrolyte networks. Following our previous study, the ionically cross-linked, LbL-assembled poly(allylamine hydrochloride)/poly(acrylic acid) (PAH/PAA, Fig. 1a) films were used as the model material. ${ }^{30}$ In aqueous solutions, the asassembled ionic PAH/PAA network is connected by ionic pairs $^{31}$ between the charged amine-groups of $\mathrm{PAH}$ (solution $\mathrm{p} K_{\mathrm{a}}$ 
a

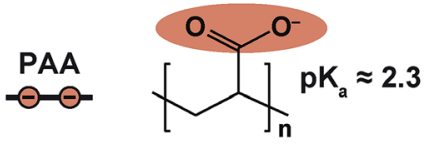

PAH

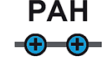

b

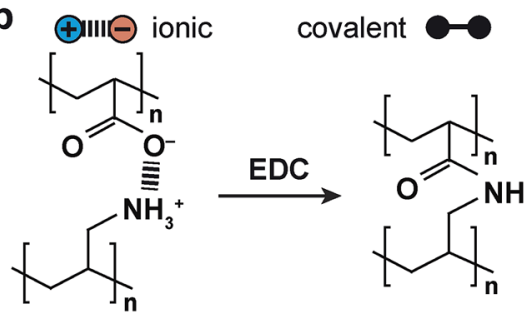

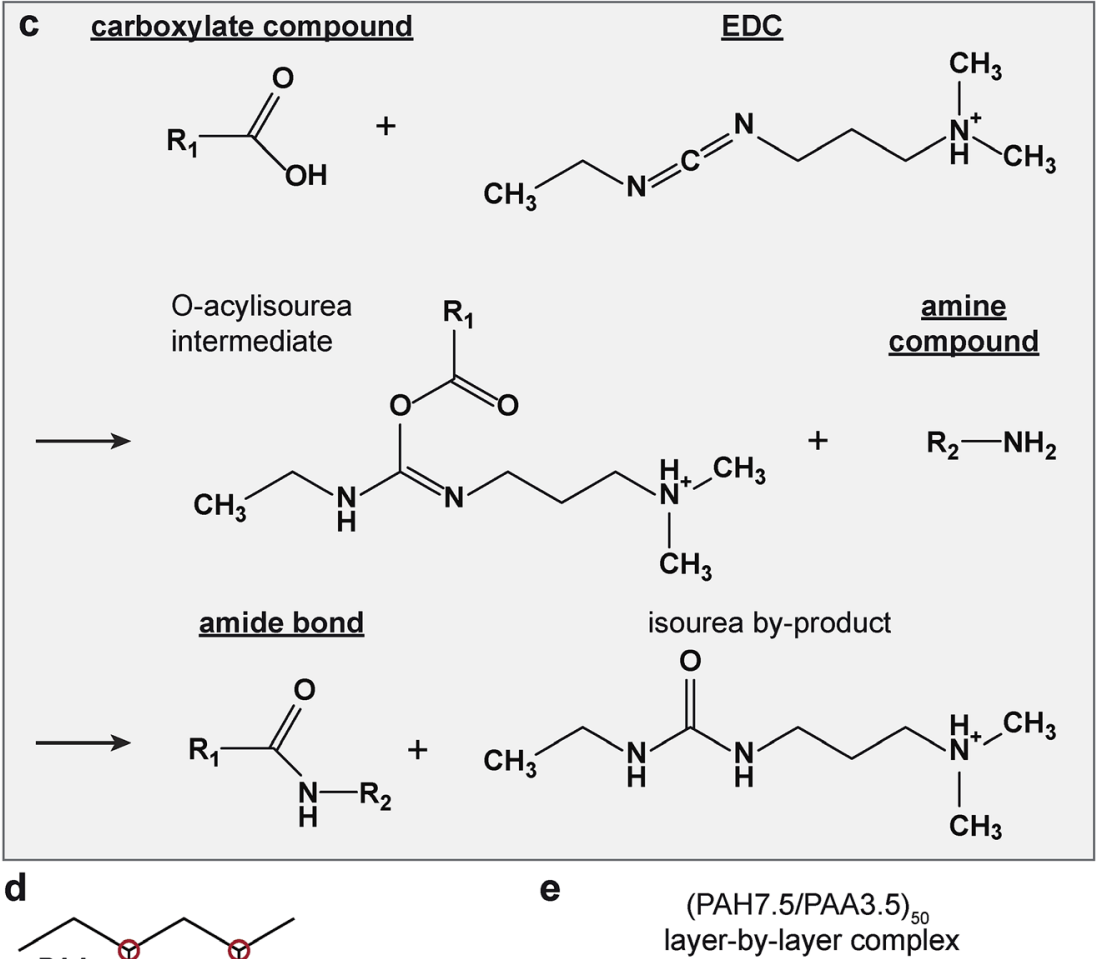
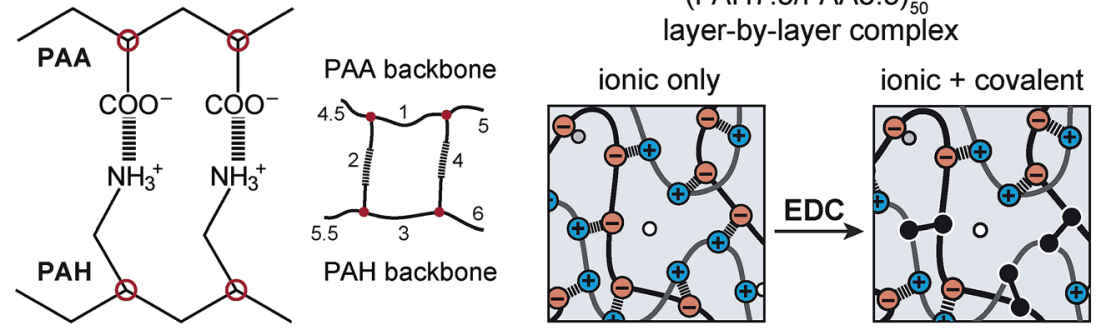

Fig. 1 Schematics of the model material system, the layer-by-layer (LbL) assembled, ionically cross-linked poly(allylamine hydrochloride)/ poly(acrylic acid) (PAH/PAA) 50 networks. (a) Molecular structure of PAH and PAA and associated pK's. (b) Impacts of EDC-treatment on the molecular structure of cross-links. (c) Chemical reactions of the formation of covalent amide bond between the carboxyl and amine groups via the carbodiimide EDC. ${ }^{36}$ (d) Schematic of the network formation via ionic cross-linking, where each pair of ionic bonding forms a network of four junctions (red circles, left panel) and six chains (numbered, right panel) according to Smith. ${ }^{37}$ (e) Schematic of the associated changes in the cross-link types of the PAH/PAA network.

$\approx 10.5)$ and the charged carboxyl groups of PAA $\left(\mathrm{p} K_{\mathrm{a}} \approx 2.3\right.$ when in the form of PAH7.5/PAA3.5 LbL films). ${ }^{32}$ Since PAH and PAA are both weak polyelectrolytes, their degrees of ionization are highly dependent on solution $\mathrm{pH}$ and ionic strength (IS). In this work, we studied the impact of additional covalent amide bonds via 1-[3-(dimethylamino)propyl]-3-ethylcarbodiimide methiodide (EDC-I) cross-linking (Fig. 1b and c) on the viscoelasticity of PAH/PAA ionic networks under the modulation of solution $\mathrm{pH}$ and IS. When the solution $\mathrm{pH}$ changes from 5.5 to $<2.0$, the degree of ionization of PAA changes from $>90 \%$ to $0 \%$, while $\mathrm{PAH}$ remains nearly fully charged. This leads to a conformational change of the PAH/PAA film, from a netneutral, highly ionically cross-linked network ( $\mathrm{pH} 5.5$ ), to a net charged, loosely cross-linked network ( $\mathrm{pH} 2.0-2.5) .^{33}$ Meanwhile, we also varied the solution IS between $0.01 \mathrm{M}$ and 1.0 $\mathrm{M}$ to introduce variations in electrostatic interactions. In net neutral states, varying IS can influence the cross-link density without affecting the charged nature of polymers through the "doping" effect. ${ }^{34}$ In net charged states, tuning IS can also affect the magnitude of Poisson-Boltzmann double layer interactions via the Debye screening. ${ }^{35}$ After the EDC treatment, each carboxyl group on PAA and each primary amine group on $\mathrm{PAH}$ 
are connected by a covalent amide bond via the carbodiimide reaction (Fig. 1c). ${ }^{36}$ As a result, the covalent amide cross-links are not affected by changes in $\mathrm{pH}$ or IS. Applying our recently developed, closed-loop nanoindentation and force relaxation tests, ${ }^{30}$ we quantified the viscoelastic properties of the PAH/PAA LbL films. These results were interpreted in the context of the PAA ionization state measured by the attenuated total reflectance-Fourier transform infrared spectroscopy (ATR-FTIR, Fig. 2a), as well as the rubber elasticity ${ }^{42}$ and its modified form of the Smith theory, ${ }^{37}$ to connect the mechanical behaviors with molecular cross-linking states. The outcomes together illustrated the distinctive viscoelastic nanomechanics of polyelectrolyte networks with two types of cross-links.

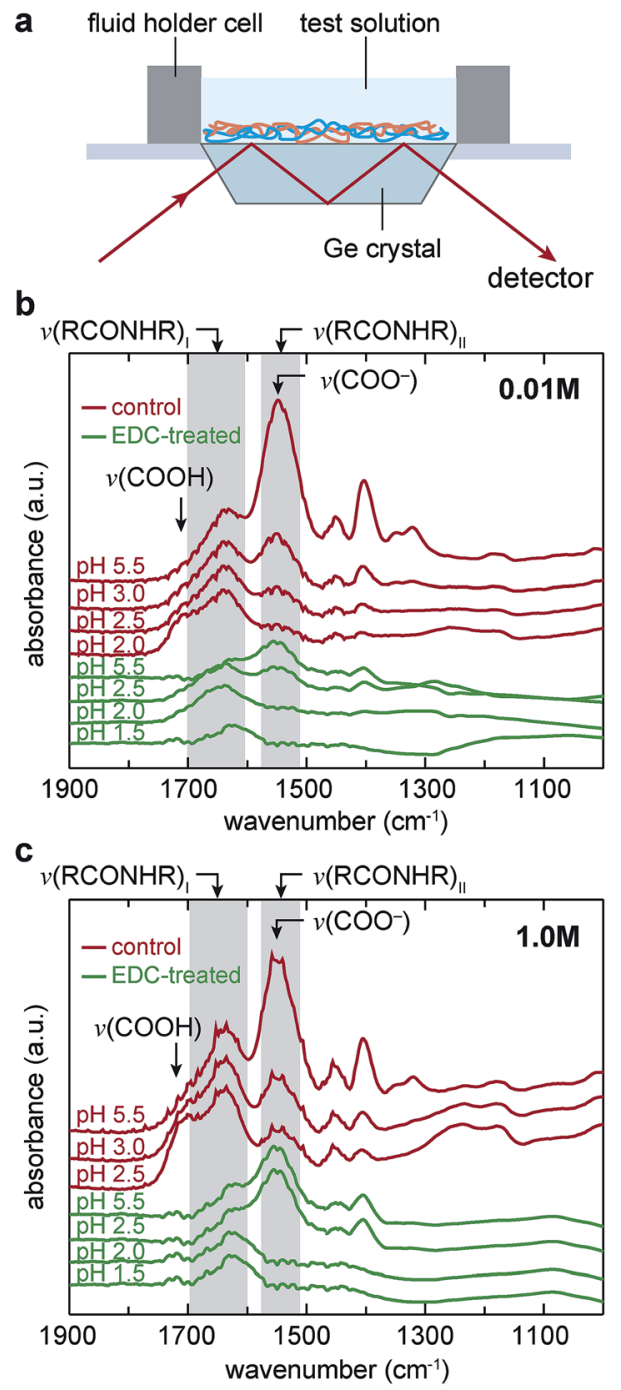

Fig. 2 ATR-FTIR test of PAH/PAA networks. (a) Schematic of the experimental set-up of measurement on the PAH/PAA films fabricated on germanium crystal, tested in aqueous solutions. (b and c) Absorbance spectra of both EDC-treated and control networks at (b) $0.01 \mathrm{M}$ and (c) $1.0 \mathrm{M} \mathrm{IS}$, respectively. Spectra are intentionally overlaid with arbitrary offset for clarity. The wavenumbers corresponding to the protonated and charged carboxyl groups, and the covalent amide bonds, are highlighted.

\section{Methods}

\subsection{Sample preparation}

Layer-by-layer assembled PAH/PAA complexes with 50-bilayers were prepared on freshly cleaned glass slides, following established procedures. ${ }^{38}$ The assembly was carried out at their partially charged states (pH 7.5 for $\mathrm{PAH}$ and $\mathrm{pH} 3.5$ for PAA) to form a relatively isotropic network with random polymer chain conformation. ${ }^{38}$ To induce additional covalent cross-linking, the LbL complexes were incubated in $\mathrm{NaCl}+\mathrm{HCl}$ aqueous solution $(0.01 \mathrm{M}, \mathrm{pH}=2.5)$ for 15 minutes, followed by $96 \mathrm{mg} \mathrm{mL}{ }^{-1} 1$-[3-(dimethylamino)propyl]-3-ethylcarbodiimide methiodide (EDC-I, corresponding to the same molar concentration of $50 \mathrm{mg} \mathrm{mL}^{-1} \mathrm{EDC}$ ) in 2-( $N$-morpholino)ethanesulfonic acid (MES) buffer for 1 hour to induce covalent amide crosslinks between PAH and PAA (Fig. 1b-e). ${ }^{39}$ The sample was then rinsed with $0.01 \mathrm{M} \mathrm{NaCl}$ solution for at least three times to remove residual reagents. For the control, the films underwent the same MES buffer incubation and rinsing procedures without the addition of EDC-I. This step was to ensure that the conformational changes of the networks due to incubation and rinsing were consistent between the control and EDC-treated groups.

\subsection{ATR-FTIR spectrometry}

To reveal the impacts of covalent cross-linking on the $\mathrm{pH}$ and ISresponsive molecular states of the carboxyl groups on PAA, ATRFTIR was applied to the PAH/PAA films prepared on a Germanium crystal puck (Specac, Orpington, United Kingdom) via the same assembly procedures. The ATR-FTIR measurement was carried out on a Nicolet Nexus 870 FT-IR Spectrometer (Thermo Fisher Scientific, Philadelphia, PA) with a Quest Single Bounce ATR accessory (Specac, Fort Washington, PA) and a custom fluid holder cell to retain the fluidic environment during the measurement (Fig. 2a), following previously reported procedures. ${ }^{40,41}$

\subsection{AFM-nanoindentation and ramp-and-hold force relaxation}

To study the elastic and viscoelastic mechanical properties, AFM-based nanomechanical tests were applied at multiple $\mathrm{pH}$ and IS to both EDC-treated and control samples, following our recently established and fully calibrated procedures. ${ }^{30}$ Briefly, AFM-nanoindentation was performed with polystyrene microspherical tips $(R \approx 12.5 \mu \mathrm{m})$ at a constant loading/unloading rate of $v_{\mathrm{D}}=5 \mu \mathrm{m} \mathrm{s}^{-1}$, up to a maximum indentation force $\sim 1 \mu \mathrm{N}$, followed by a $\sim 30 \mathrm{~s}$ force relaxation holding period via the indenter mode of the Molecular Force Probe 3D (Asylum Research, Santa Barbara, CA). In this closed-loop mode, the cantilever displacement was continuously adjusted to compensate for the time-dependent viscoelastic softening of the sample, resulting in constant indentation rate and constant indentation depth during relaxation. These tests were performed in $\mathrm{pH}=1.5-5.5, \mathrm{IS}=0.01 \mathrm{M}$ and $1.0 \mathrm{M} \mathrm{NaCl}+\mathrm{HCl}$ aqueous solutions, which modulated the degree of ionic crosslinking and fixed charge-governed electrostatic double-layer 
interactions. At each IS and $\mathrm{pH}$, the test was repeated on 3 samples, $\geq 10$ locations each sample, and no significant differences were found amongst different samples. At each state, the thickness of the film, $H$, was measured by AFM via selective film removal followed by contact mode AFM imaging for $H \leq 10 \mu \mathrm{m}$, and our recently developed $z$-motor assisted method for $H>10 \mu \mathrm{m} .^{30}$

With a maximum indentation depth $D_{\max }<2 \mu \mathrm{m}$, the maximum strain was controlled at less than $20 \%$ to ensure that the deformation of PAH/PAA was in the linear regime. Linear elastic and viscoelastic models were applied to extract material mechanical properties. The effective indentation modulus, $E_{\text {ind }}$, was calculated via the Hertz model with finite thickness correction, ${ }^{42}$

$$
F=\frac{4}{3} \frac{E_{\text {ind }}}{\left(1-\nu_{\mathrm{P}}^{2}\right)} R_{\text {tip }}{ }^{1 / 2} D^{3 / 2} C_{\chi}\left(F, D, H, R_{\text {tip }}\right),
$$

where $\nu_{\mathrm{P}}$ is the Poisson's ratio $(\approx 0.49$ for highly swollen hydrogels), ${ }^{43} R_{\text {tip }}$ is the tip radius, and $C_{\chi}$ is the substrate constraint correction factor that depends on $F, D, H$ and $R_{\text {tip }}$. To estimate the time-dependent mechanical properties, at each time point, the temporal modulus, $E(t)$, was calculated by fitting $F(t)$ and $D$ to the substrate-corrected Hertz model, whereby $D$ was constant during the relaxation. The relaxation of, $E(t)$, was then fitted to a five-element standard linear solid (SLS) model ${ }^{\mathbf{4 4}}$ to extract two time constants $\tau_{1}$ and $\tau_{2}$,

$$
E(t)=E_{\infty}+E_{1} \exp \left(-t / \tau_{1}\right)+E_{2} \exp \left(-t / \tau_{2}\right),
$$

Here, $E_{\infty}$ is the equilibrium indentation modulus, $E_{1}$ and $E_{2}$ are the time-dependent modulus corresponding to the short and long term relaxation mode $\left(\tau_{1}<\tau_{2}\right)$, respectively. The instantaneous indentation modulus, $E_{0}$, was estimated as, $E_{0}=$ $E_{\infty}+E_{1}+E_{2}$. We used the ratio of $E_{\infty} / E_{0}$ as a measure of the degree of elasticity.

\subsection{Estimation of effective cross-link density}

To understand the contributions of covalent and ionic crosslinks to the mechanical properties, we estimated the effective cross-link densities of both cross-link types via the classical rubber elasticity theory ${ }^{45}$ and its extended Smith theory. ${ }^{37}$ For covalent cross-links, the effective density was estimated by applying the linear rubber elasticity theory to the equilibrium modulus, $E_{\infty}$, measured at $\mathrm{pH} 1.5$, IS $=1.0 \mathrm{M}$. At this state, the low $\mathrm{pH}$ ensures full protonation of carboxyl groups, and thus, absence of ionic cross-linking, and the high IS results in full screening of the Donnan effect. Therefore, $E_{\infty}$ is dominated by the rubber elasticity from covalent cross-links. The use of $E_{\infty}$, instead of the indentation modulus $E_{\text {ind }}$, also minimized the possible overestimation from the effect of intrinsic viscoelasticity or poroelasticity. The effective polymer chain density (molar number of chains per unit volume), $\nu$, was calculated as,

$$
E_{\infty}=3 \phi k_{\mathrm{B}} T \nu
$$

where $\phi$ is a front factor associated with polymer chain configuration $(\approx 0.5),{ }^{37,46} k_{\mathrm{B}}$ is the Boltzmann constant, and $T$ is the temperature in $\mathrm{K}$. Following the rubber elasticity theory, $\nu$ at other states were estimated using the ratio of volume calculated from the thickness measurement. Following the definition by Smith, ${ }^{37}$ for every two cross-links, there exist four network junctions and six chains (Fig. 1c), and the internal amide bond density, $c_{\mathrm{ec}}$, is given by,

$$
\nu=3 c_{\mathrm{ec}}
$$

For ionically cross-linked networks, the effective ionic crosslink density, $c_{\mathrm{ei}}$, cannot be estimated with the same method, due to two major reasons. First, rubber elasticity is limited to loose networks where the number of monomer units between adjacent links is $\geq 10 .{ }^{46,47}$ This assumption is valid for covalent networks. However, for ionically cross-linked networks, especially in netneutral conditions, where all charged groups can potentially serve as cross-linking sites, this assumption does not hold. The Smith theory ${ }^{37}$ was thus used to calculate the cross-link density $\nu$,

$$
3 \nu k_{\mathrm{B}} T / E=\left(1 / \phi_{n}\right)-6 \bar{C}_{n} /\left(5 q^{2} \bar{n}\right)
$$

where $E$ is the elastic modulus, $q$ is a dimensionless factor determined by bond angles and lengths $(q=0.83$ for chains containing only carbon-carbon single bonds, as in PAA and $\mathrm{PAH}), \bar{C}_{n}$ is a characteristic ratio equals $\bar{n}^{0.57}$, and $\phi_{n}$ is the front factor $(0.5$ for $\bar{n}>9.6)$. The term, $\bar{n}$, is the geometric mean number of backbone bonds per chain, calculated as

$$
\bar{n}=\left[\left(\frac{n_{\mathrm{PAH}}}{(1-y) \Omega}\right)\left(\frac{n_{\mathrm{PAA}}}{(1-y) \Omega}\right) n_{\text {ion pair }}\right]^{1 / 3},
$$

where $n_{\mathrm{PAH}}, n_{\mathrm{PAA}}$ and $n_{\text {ion pair }}$ are the number of bonds in the PAA, PAH and ionic pairs at the highest possible cross-linking (2, 2 and 4 respectively), $y$ is relative degree of doping level as a result of extrinsic ionic pairing $(y \approx 0.035$ and 0.31 at $0.01 \mathrm{M}$ and $1.0 \mathrm{M}$, respectively) and $\Omega$ is the fraction of ion pairing in the network mode $(\approx 0.5) \cdot{ }^{37,46}$ Similar to the case of covalent networks, the intrinsic ion pairing density that contributes to the network cross-linking is, $\nu=3 c_{\mathrm{ei}}$.

Second, the viscoelastic relaxation process of ionically crosslinked network is dominated by the local breaking and reformation of ionic cross-links. ${ }^{30}$ Therefore, unlike the case of covalent networks, $E_{\infty}$ here does not reflect the effective ionic cross-link density at the un-deformed state. The instantaneous indentation modulus, $E_{\text {ind }}$, on the other hand, was measured before the cross-link breaking or re-formation had taken place, and thus, could better reflect the ionic cross-link density at the un-deformed state. Under this scenario, the use of $E_{\text {ind }}$ may over-estimate $c_{\mathrm{ei}}$, as it does not exclude the viscoelastic and poroelastic contributions from polymer chains conformational changes and interstitial fluid flow. However, as we demonstrated in our previous work, cross-link breaking and reformation is the dominating viscoelasticity mechanism in ionic networks. ${ }^{30}$ These other effects are expected to be minor, and thus, $E_{\text {ind }}$, rather than $E_{\infty}$, was used to estimate $c_{\text {ei }}$. Further, it should be noted that, even in the duration of indentation, ionic cross-links can break and re-form, the use of $E_{\text {ind }}$ is an approximation, rather than an accurate estimate of $c_{\mathrm{ei}}$. 
We applied eqn (4) to both the control and EDC-treated networks at $\mathrm{pH} 2.5-5.5$, IS $=0.01 \mathrm{M}$ and $1.0 \mathrm{M}$. For the control networks that were cross-linked solely by ionic pairing, this calculation directly yielded the intrinsic ionic pairing density, $c_{\text {ei }}$. For the EDC-treated networks, the application of eqn (4) yields an effective net cross-link density, $c_{\text {et }}$. This effective density includes the densities of both ionic pairing and amide bonding, i.e., $c_{\mathrm{et}}=c_{\mathrm{ec}}+c_{\mathrm{ei}}$. Here, the effective amide bonding density, $c_{\mathrm{ec}}$, was estimated using $E_{\infty}$ measured at $\mathrm{pH}$ 1.5 , IS $=1.0 \mathrm{M}$ and considering that the covalent cross-links remain unchanged, the $c_{\mathrm{ec}}$ at each condition can be estimated with the rubber elasticity theory with volume change calculated from the changes in film thickness $H$. Therefore, the effective ionic pairing density can be deduced as, $c_{\mathrm{ei}}=c_{\mathrm{et}}-c_{\mathrm{ec}}$. Here, the outcome of effective density only represents the bondings that contribute to the formation of polymer chain networks. Those not contributing to the network formation, such as the "ladder" bonding, ${ }^{46}$ do not contribute to the modulus, and thus, were not taken into account by this calculation.

\section{Results}

\section{1 pH-dependent charging and protonation of carboxyl groups on PAA via ATR-FTIR}

The ATR-FTIR results revealed a clear trend of pH-dependent charging and protonation of the carboxyl acid groups on PAA (Fig. $2 \mathrm{~b}$ and c). The protonated carboxyl group $(-\mathrm{COOH})$ absorbs infrared light at wavenumber around $1710-1700 \mathrm{~cm}^{-1}$ and the charged carboxyl group $\left(-\mathrm{COO}^{-}\right)$at $1565-1542 \mathrm{~cm}^{-1} \cdot{ }^{33}$ Since other chemical bonds have minimum absorbance at this range, we focused our analysis on the wavenumber range between $1900-1300 \mathrm{~cm}^{-1}$ to illustrate the degree of ionization of the carboxyl groups on PAA. Moreover, since the absorbance peaks between $-\mathrm{NH}_{3}{ }^{+}$and $-\mathrm{CH}_{2}$ groups are overlapping near $3000 \mathrm{~cm}^{-1}$ wavenumber, ${ }^{33}$ and amine groups were maintained at near fully charged state throughout this study, we did not analyze the degree of ionization for $\mathrm{PAH}$.

For both the control and EDC-treated networks, at both IS of $0.01 \mathrm{M}$ and $1.0 \mathrm{M}$, changing the solution $\mathrm{pH}$ from 5.5 to 2.0 results in a substantial decrease in the peak height at the wavenumber of $\nu\left(\mathrm{COO}^{-}\right)=1565-1542 \mathrm{~cm}^{-1},{ }^{33}$ demonstrating the protonation of $\mathrm{COO}^{-}$as $\mathrm{pH}$ changes past its $\mathrm{p} K_{\mathrm{a}}$. At the same time, there was an increase in the peak height at the wavenumber of $\nu(\mathrm{COOH})=1710-1700 \mathrm{~cm}^{-1},{ }^{33}$ which was less pronounced in comparison to the decrease of the peak height at $\nu\left(\mathrm{COO}^{-}\right)$. This is because decreasing $\mathrm{pH}$ results in swelling of PAH/PAA, and thus, decreases the concentration of polymer chains that is probed by ATR-FTIR. This effect attenuates the effect of increased carboxyl protonation to the peak at $\nu(\mathrm{COOH})$, and amplifies the effect of decreased to the peak at $\nu\left(\mathrm{COO}^{-}\right)$.

The trend of $\mathrm{pH}$-dependence was consistent for the control and EDC-treated networks, indicating that added covalent cross-linking does not significantly alter the $\mathrm{pH}$-responsiveness of the PAH/PAA ionic complex. Meanwhile, the peak at $\nu\left(\mathrm{COO}^{-}\right)$ for the EDC-treated network was also less pronounced than the control, which can be attributed to the larger degree of swelling, and thus, lower polymer volume concentration (Fig. 3a).

In theory, EDC-induced cross-linking results in amide bonds (-CONH-), which absorbs infrared light at wavenumber $\sim 1700-$ 1600 and $1580-1510 \mathrm{~cm}^{-1} \cdot{ }^{48}$ However, as shown by the results for EDC-treated network at $\mathrm{pH} 1.5$, when all carboxyl groups are protonated, we did not observe significant increase at this wavenumber (Fig. $2 \mathrm{~b}$ and c). This suggests that the overall molar ratio of amide bonds is very small compared to the number of free carboxyl acid groups, and its presence does not obscure our observation on $\mathrm{pH}$-induced carboxyl protonation.

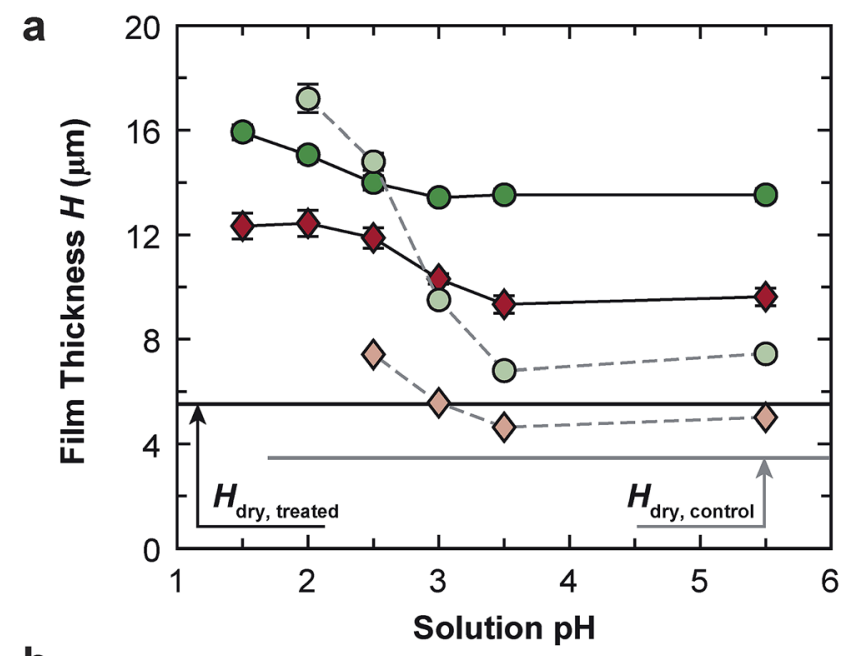

b

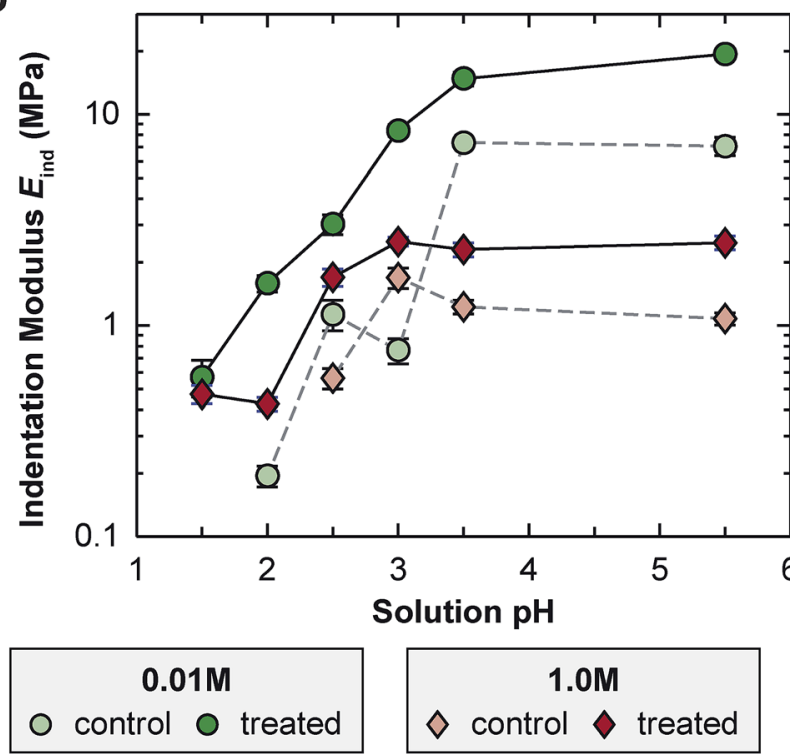

Fig. 3 Impacts of covalent cross-linking on the film thickness, $H$, and effective indentation modulus of (PAH7.5/PAA3.5) 50 LbL networks at various $\mathrm{pH}$ and IS. (a) Film thickness, $H$, at all tested $\mathrm{pH}$ and IS (mean \pm SEM for $n \geq 15$ measurements on at least three samples at each condition). The dashed line represents the dry film thickness (SEM is less than the line width). (b) Instantaneous indentation modulus calculated from $F-D$ curves, $E_{0}$, of (PAH7.5/PAA3.5) $)_{50}$ at various $\mathrm{pH}$ and IS (mean \pm SEM for $n \geq 15$ positions on at least three samples). All experiments were performed at $5 \mu \mathrm{m} \mathrm{s}^{-1}$ constant indentation depth rate by a microspherical tip $(R \approx 12.5 \mu \mathrm{m})$. 


\subsection{Swelling and stability of PAH/PAA}

AFM-thickness measurement highlighted the differences in the swelling between EDC-treated and control networks (Fig. 3a and Table 1). At the low IS of $0.01 \mathrm{M}$ (Debye length $\kappa^{-1} \approx 3 \mathrm{~nm}$ ), for the control network, the film thickness, $H$, increased from $7.4 \pm$ $0.1 \mu \mathrm{m}$ (mean $\pm \mathrm{SEM}, n \geq 15$ ) to $17.2 \pm 0.5 \mu \mathrm{m}$ when changing $\mathrm{pH}$ from 5.5 to 2.0 , a $2.31 \pm 0.07 \times$ difference; for the EDCtreated one, $H$ increased from $13.5 \pm 0.2 \mu \mathrm{m}$ to $15.0 \pm$ $0.2 \mu \mathrm{m}$, a $1.12 \pm 0.03 \times$ difference. This pronounced swelling for both networks was due to the Donnan effect arising from net positive charges on PAH as PAA becomes protonated. The contrast between the control and EDC-treated ones highlighted that the presence of permanent anchoring by covalent crosslinks can effectively reduce this Donnan effect-induced swelling. At the high ionic strength of $1.0 \mathrm{M}$, when Donnan effect is fully screened by the free counter ions $\left(\kappa^{-1} \approx 0.3 \mathrm{~nm}\right)$, reducing $\mathrm{pH}$ leads to less swelling for both networks.

The EDC-treated network had greater $H$ than the control at nearly all states except for the highly swollen one. While we do not fully understand the mechanism behind the increased film thickness after EDC treatment, this difference likely suggests possible conformational changes of the network due to EDCinduced covalent cross-linking, which has been shown to take place for LbL poly(L-lysine)/poly(L-glutamic acid) (PLL/PGA) nanofilms. ${ }^{49}$ However, we do not expect the lower thickness of control film, especially at the dry state, to reflect appreciable material loss during the step of 2-( $N$-morpholino)ethanesulfonic acid (MES) immersion. This is because the control, MES-immersed network here showed highly similar swelling and mechanical properties to our previously reported results on as-prepared PAH/PAA films, which did not undergo any solution treatment $\left(\right.$ e.g., $E_{\text {ind }}=8.3 \pm 0.8 \mathrm{MPa}$, and $7.1 \pm 0.7 \mathrm{MPa}$ before and after MES immersion ( $p>0.05)$, and $H=7.1 \pm 0.1 \mu \mathrm{m}$, and $7.4 \pm 0.1 \mu \mathrm{m}$ before and after immersion, when measured at $\mathrm{pH}$ $5.5,0.01 \mathrm{M}){ }^{30}$ We also do not expect appreciable reaction byproduct entrapped in the EDC-treated network, since ATRFTIR did not detect the peak associated with the alkyl form of iso-urea bonding (wavenumber $\sim 1200-1025 \mathrm{~cm}^{-1}$, 50 Fig. $2 \mathrm{~b}$ and $\mathrm{c})$.

When $\mathrm{pH}$ was lowered to 1.5 , the control network was dissolved upon complete loss of ionic cross-links, while the EDCtreated network remains stable. At this state, the network is held solely by covalent cross-links, and the greater swelling at $0.01 \mathrm{M}$ than $1.0 \mathrm{M}$ reflects the effects of fixed charge-induced Donnan osmotic effect. ${ }^{35}$

\subsection{Elastic and viscoelastic nanomechanical properties}

AFM-nanoindentation detected significantly higher modulus for the EDC-treated network at all tested conditions (Fig. $3 \mathrm{~b}$ and Table $1 \mathrm{a}-\mathrm{c}$ ), e.g., $E_{\text {ind,EDC }} / E_{\text {ind,control }}=3.0 \pm 0.3$ at $\mathrm{pH}=5.5$, $0.01 \mathrm{M}$ and $2.4 \pm 0.2$ at $\mathrm{pH}=5.5,1.0 \mathrm{M}$. However, covalent cross-linking did not substantially alter the stimulus responses of $E_{\text {ind }}$ to solution pH and IS. At $0.01 \mathrm{M}$ IS, reducing pH from 5.5 to 2.5 resulted in a $7.3 \pm 1.3$ fold and $7.4 \pm 0.8$ fold decrease in $E_{\text {ind }}$ for the control and EDC-treated networks, respectively. This trend was similar at 1.0 M IS, in which, the modulus decreases
Table 1 Swelling and nanomechanical properties of (PAH7.5/ PAA3.5) 50 networks at various cross-link and fixed charge densities. $(\mathrm{a}-\mathrm{c})$ Comparison between the EDC-treated and control networks at three representative cross-link and fixed charge density states. (d) Comparison of the solely covalently cross-linked network $(\mathrm{pH} 1.5)$ at different ionic strengths. The fold of changes were calculated only when Mann-Whitney $U$ test detected significant differences $(p<0.05)$, and n.s. indicates no statistical differences $(p>0.05)$. All the results are presented as mean \pm SEM from $n \geq 15$ measurements

a net neutral, highly ionically linked $(\mathrm{pH} 5.5,0.01 \mathrm{M})$

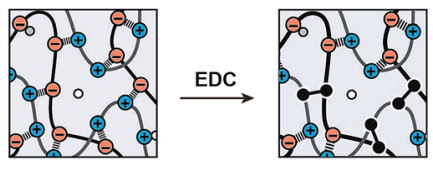

\begin{tabular}{lccc}
\hline & Control & Treated & Fold change \\
\hline$H(\mu \mathrm{m})$ & $7.4 \pm 0.1$ & $13.5 \pm 0.2$ & $1.8 \pm 0.1$ \\
$E_{\text {ind }}(\mathrm{MPa})$ & $7.1 \pm 0.7$ & $19.4 \pm 1.1$ & $3.0 \pm 0.3$ \\
$E_{\infty} / E_{0}(\%)$ & $3.8 \pm 1.0$ & $20.0 \pm 2.7$ & $6.3 \pm 1.8$ \\
$\tau_{1}(\mathrm{~s})$ & $0.061 \pm 0.002$ & $0.097 \pm 0.011$ & $1.6 \pm 0.3$ \\
$\tau_{2}(\mathrm{~s})$ & $3.5 \pm 0.2$ & $4.5 \pm 0.5$ & $1.3 \pm 0.3$ \\
\hline
\end{tabular}

b net neutral, less ionically linked ( $\mathrm{pH} 5.5,1.0 \mathrm{M})$

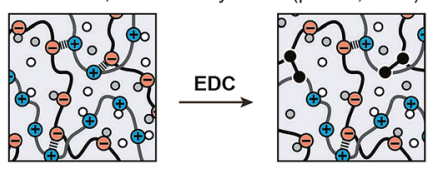

\begin{tabular}{lccc}
\hline & Control & Treated & Fold change \\
\hline$H(\mu \mathrm{m})$ & $5.0 \pm 0.1$ & $9.6 \pm 0.3$ & $1.9 \pm 0.1$ \\
$E_{\text {ind }}(\mathrm{MPa})$ & $1.1 \pm 0.1$ & $2.5 \pm 0.2$ & $2.4 \pm 0.3$ \\
$E_{\infty} / E_{0}(\%)$ & $1.4 \pm 0.6$ & $32.8 \pm 2.4$ & $44 \pm 10$ \\
$\tau_{1}(\mathrm{~s})$ & $0.047 \pm 0.002$ & $0.101 \pm 0.019$ & $2.2 \pm 0.4$ \\
$\tau_{2}(\mathrm{~s})$ & $1.1 \pm 0.1$ & $4.7 \pm 0.8$ & $4.6 \pm 1.0$ \\
\hline
\end{tabular}

c net charged, least ionically linked $(\mathrm{pH} 2.0,0.01 \mathrm{M})$

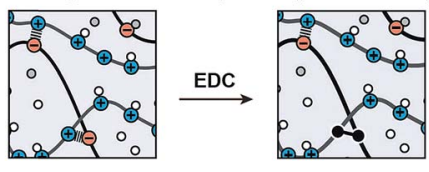

\begin{tabular}{lccl}
\hline & Control & Treated & Fold change \\
\hline$H(\mu \mathrm{m})$ & $17.2 \pm 0.5$ & $15.0 \pm 0.2$ & $0.9 \pm 0.1$ \\
$E_{\text {ind }}(\mathrm{MPa})$ & $0.19 \pm 0.02$ & $1.59 \pm 0.14$ & $8.9 \pm 1.5$ \\
$E_{\infty} / E_{0}(\%)$ & $40.2 \pm 2.1$ & $55.1 \pm 2.8$ & $1.4 \pm 0.1$ \\
$\tau_{1}(\mathrm{~s})$ & $0.132 \pm 0.011$ & $0.103 \pm 0.017$ & n.s. \\
$\tau_{2}(\mathrm{~s})$ & $5.0 \pm 0.2$ & $5.0 \pm 1.5$ & n.s. \\
\hline
\end{tabular}

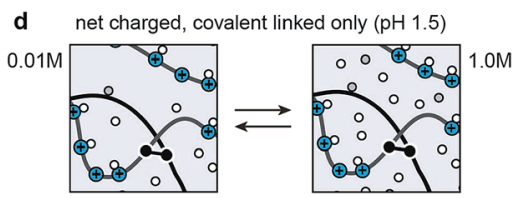

\begin{tabular}{lccl}
\hline & $0.01 \mathrm{M}$ & $1.0 \mathrm{M}$ & Fold change \\
\hline$H(\mu \mathrm{m})$ & $15.9 \pm 0.3$ & $12.3 \pm 0.5$ & $0.78 \pm 0.03$ \\
$E_{\text {ind }}(\mathrm{MPa})$ & $0.57 \pm 0.11$ & $0.47 \pm 0.05$ & $0.83 \pm 0.02$ \\
$E_{\infty} / E_{0}(\%)$ & $69.0 \pm 1.6$ & $71.5 \pm 2.3$ & n.s. \\
$\tau_{1}(\mathrm{~s})$ & $0.080 \pm 0.021$ & $0.090 \pm 0.027$ & n.s. \\
$\tau_{2}(\mathrm{~s})$ & $5.7 \pm 1.2$ & $3.1 \pm 0.6$ & n.s.
\end{tabular}


by $2.1 \pm 0.3$ fold and $1.7 \pm 0.2$ fold for the control and EDCtreated networks, respectively, from $\mathrm{pH} 5.5$ to 2.5 . On the other hand, at $\mathrm{pH} 5.5$, changing IS from $0.01 \mathrm{M}$ to $1.0 \mathrm{M}$ resulted in a $7.0 \pm 0.9 \times$ and $8.7 \pm 0.9 \times$ decrease in $E_{\text {ind }}$ for the control and EDC-treated ones, respectively. In addition, when $\mathrm{pH}$ was lowered to 1.5 , while the control was dissolved upon complete loss of ionic cross-links, the EDC-treated network remained stable, and increasing IS from $0.01 \mathrm{M}$ to $1.0 \mathrm{M}$ only decreased $E_{\text {ind }}$ for $17 \pm 2 \%$.

Closed-loop force relaxation test yielded significantly different viscoelastic behaviors (Fig. 4a). For the control, a near $100 \%$ relaxation was observed at all net neutral states $(\mathrm{pH} 2.5-$ $5.5,0.01-1.0 \mathrm{M}$ ), in which, as noted by our previous study, the relaxation is dominated by ionic cross-link breaking and reformation. $^{30}$ Adding the permanent, covalent cross-links increased the degree of elasticity $\left(E_{\infty} / E_{0}\right)$, and in turn, decreased the degree of relaxation $\left(1-E_{\infty} / E_{0}\right)$ (Fig. 4b). For example, at pH 5.5, 0.01 M, the degree of relaxation was $96 \pm 1 \%$ for the control, and was reduced to $80 \pm 3 \%$ for the EDC-treated network. At the highly swollen state of $\mathrm{pH} 2.0,0.01 \mathrm{M}$, when additional elasticity was endowed by the Donnan effect, $E_{\infty} / E_{0}$ was $40 \pm 2 \%$ for the control, and the treatment further increased the elasticity, $E_{\infty} / E_{0}=55 \pm 3 \%$.

Since adding covalent cross-links increased the degree of elasticity $\left(E_{\infty} / E_{0}\right)$, as expected, the contribution of the two viscoelastic modes, the short-term relaxation $\left(E_{1}, \tau_{1}\right)$ and longterm relaxation $\left(E_{2}, \tau_{2}\right)$ (see Experimental section for more details), to the overall modulus were reduced at all tested states (Fig. 5a and $\mathrm{b}$ and Table $1 \mathrm{a}-\mathrm{c}$ ). At all net neutral states ( $\mathrm{pH}$ 2.5-5.5, 0.01 and $1.0 \mathrm{M}$ ), adding cross-links significantly increased both the short term, $\tau_{1}$, and the long term, $\tau_{2}$, relaxation time constants (Fig. 5c and $\mathrm{d}$ and Table $1 \mathrm{a}$ and $\mathrm{b}$ ). At the net charged state $(\mathrm{pH} 2.0,0.01 \mathrm{M})$, on the other hand, the cross-linked state had similar $\tau_{1}$ and $\tau_{2}$ compared to the control (Fig. $5 \mathrm{c}$ and d, Table $1 \mathrm{c}$ ).

\subsection{Covalent and ionic cross-link density estimation}

Based on classical rubber elasticity theory, ${ }^{45}$ at $\mathrm{pH} 1.5$, IS $=$ 1.0 M, the effective covalent cross-link density, $c_{\mathrm{ec}}$, was calculated to be $91 \pm 33 \mathrm{~mol} \mathrm{~m}^{-3}$, or $0.09 \pm 0.03 \mathrm{M}$. Values of $c_{\mathrm{ec}}$ at other states, which were estimated based on the swelling ratio, did not undergo substantial changes (Fig. 6). For the control network, in accordance with the $\mathrm{pH}$ - and IS-responsiveness of $E_{\text {ind }}$, the effective ionic cross-link density, $c_{\text {ei }}$, also showed significant changes with $\mathrm{pH}$ and IS. For the EDC-treated network containing both covalent and ionic cross-links, the effective total cross-link density, $c_{\text {et }}$, was higher than that of the control network at all tested states (Fig. 6a). Furthermore, after subtracting the covalent cross-link density, the effective ionic cross-link density still appears higher than that of the control network (Fig. 6b). These results highlighted a synergistic, non-

a
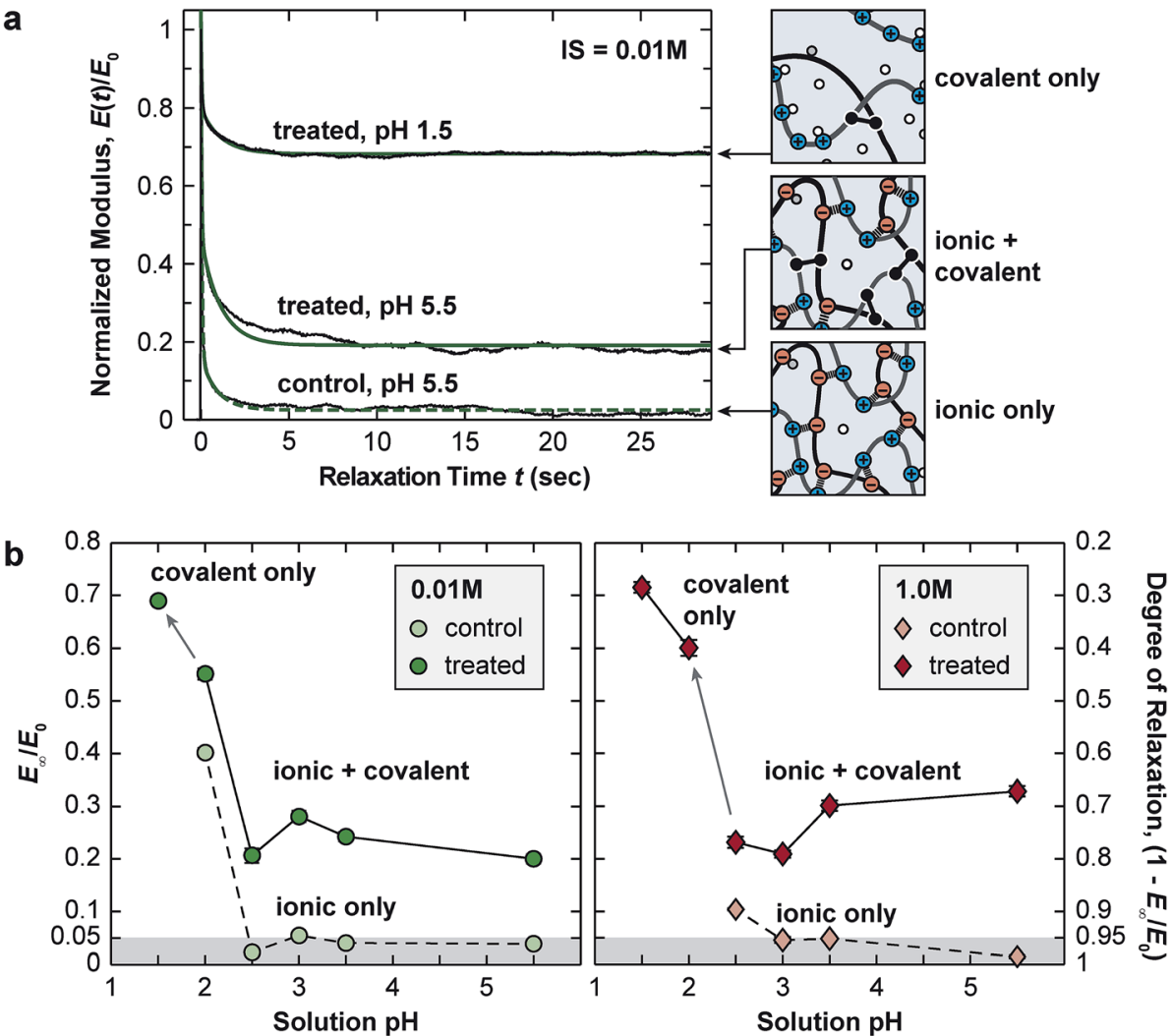

Fig. 4 Impacts of covalent cross-linking on viscoelastic relaxation of (PAH7.5/PAA3.5) 50 LbL network at various pH and IS. (a) Representative normalized $E(t)$ versus initial indentation modulus, $E_{0}$, relaxation curves showing distinctive relaxation behaviors in the presence of covalent cross-links. Dashed lines are the SLS model fit by non-linear least squares regression. (b) The degree of elasticity, $E_{\infty} / E_{0}$, and the associated degree of relaxation, $\left(1-E_{\infty} / E_{0}\right)$ at all tested $\mathrm{pH}$ and IS (mean $\pm \mathrm{SEM}, n \geq 15, *: p<0.05$ via Mann-Whitney $U$ test). 
a

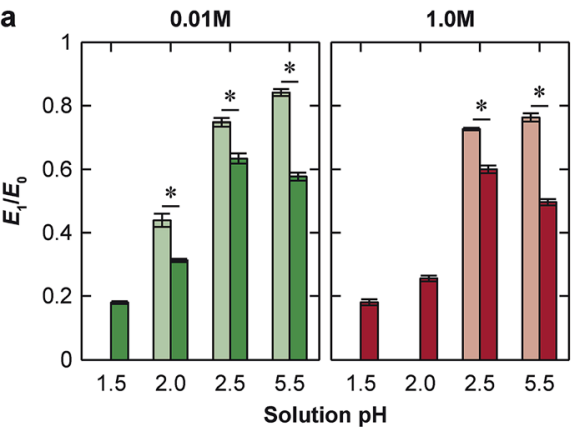

c

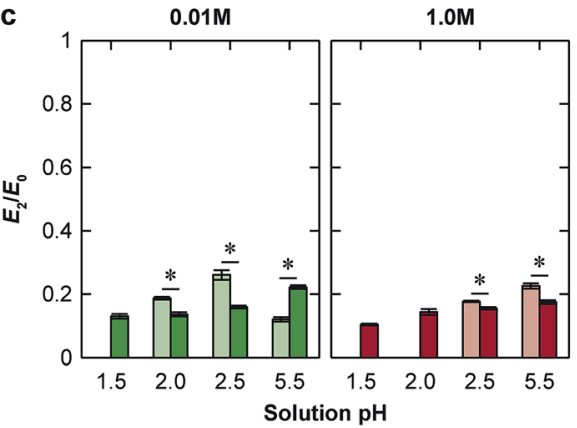

b

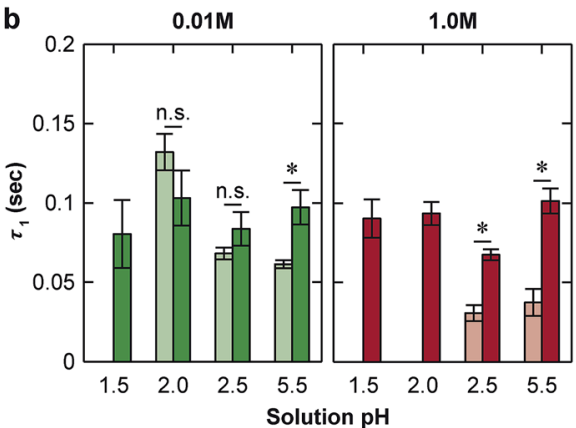

d

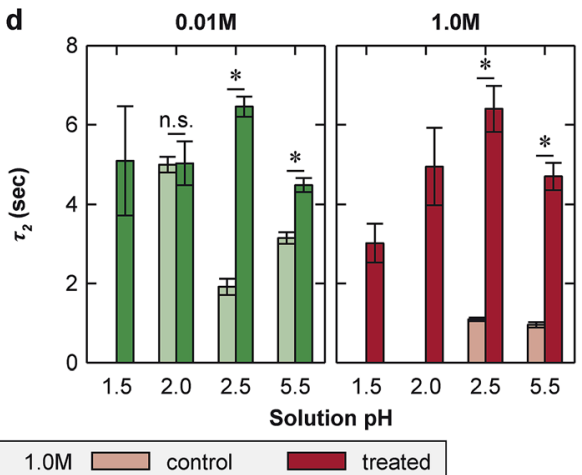

Fig. 5 Impacts of covalent cross-linking on the viscoelastic relaxation at both shorter $(\mathrm{a}, \mathrm{b})$ and longer (c, d) times at different $\mathrm{pH}$ and IS, predicted by the five-element SLS model. (a) $E_{1}$ and (c) $E_{2}$ normalized by initial modulus, $E_{0}$, and corresponding time constants (b) $\tau_{1}$ and (d) $\tau_{2}$ (mean \pm SEM, $n \geq 15, *: p<0.05$ via Mann-Whitney $U$ test).
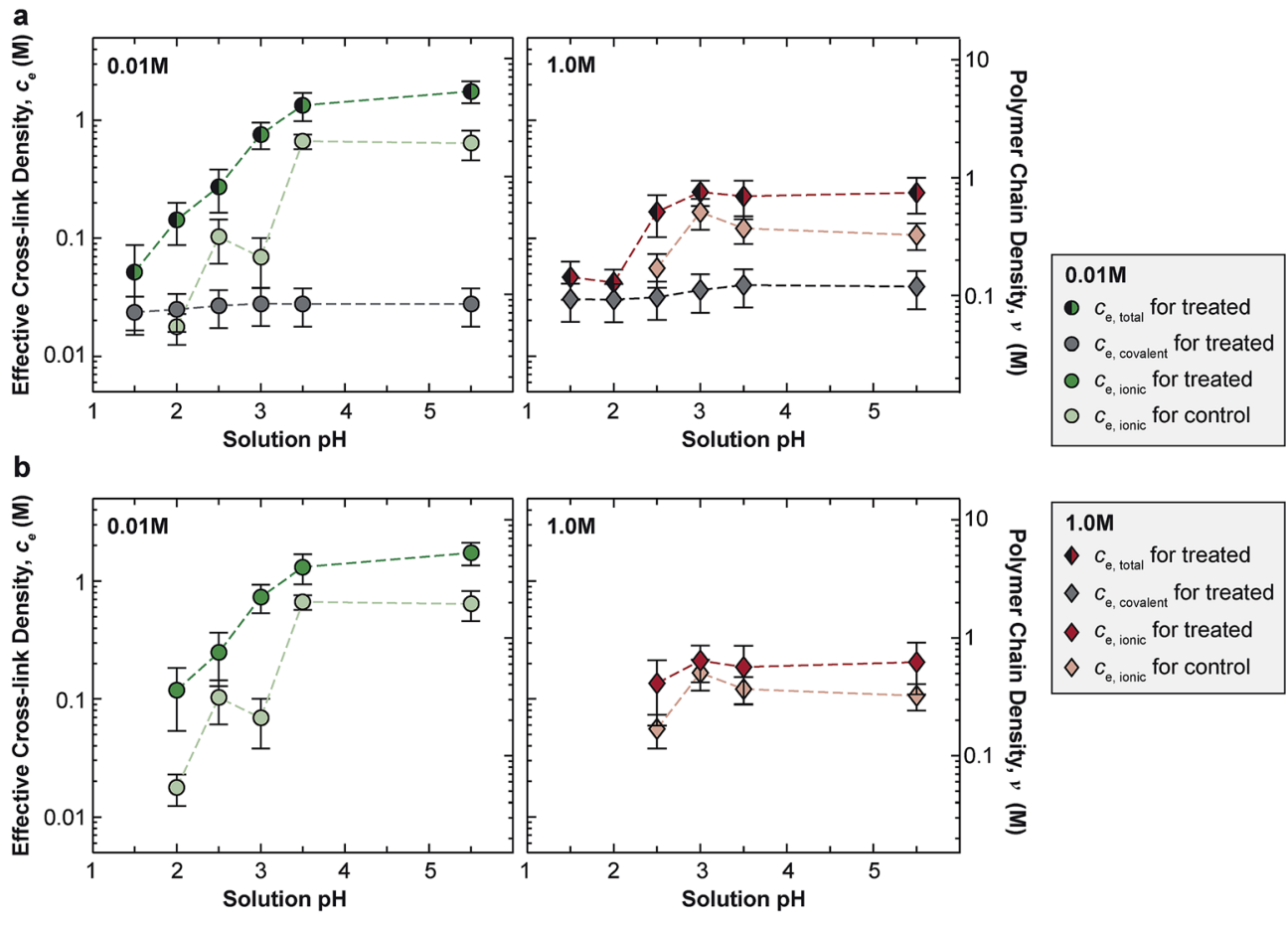

Fig. 6 Comparison of effective cross-link densities estimated based on the classic rubber elasticity theory and the Smith theory for tightly crosslinked networks. Both the densities of effective cross-link bonding (intrinsic carboxyl-amine pairing for ionic, and EDC-induced amide bonding for covalent) are plotted. (a) Comparison between the total cross-link density and the covalent cross-link density of EDC-treated work, versus the ionic cross-link density of the control at both $0.01 \mathrm{M}$ and 1.0 M IS. (b) Comparison between the ionic cross-link density between the EDC-treated and control groups at both IS (mean \pm SEM, $n \geq 15$ ). 
additive impact between the covalent and ionic cross-links. The additional covalent cross-links can change the chain conformation of PAH and PAA, which likely affects the equilibrium state of the ionic network, leading to an increase in ionic crosslinking density.

\section{Discussion}

4.1 Viscoelasticity at net neutral states: non-additive impacts of covalent cross-linking

While covalent cross-linking is expected to increase $E_{\text {ind }}$ (Fig. 3b), the magnitude of change does not scale with the covalent cross-link density in an additive manner. For example, at $\mathrm{pH} 5.5,0.01 \mathrm{M}, E_{\text {ind }}$ was $7.1 \pm 0.7 \mathrm{MPa}$ for the control, and 19.4 $\pm 1.1 \mathrm{MPa}$ for the EDC-treated network, yielding a difference of $12.3 \pm 1.8 \mathrm{MPa}$ (Table 1a). By contrast, at this state, given the covalent cross-link density of $83 \pm$ $30 \mathrm{~mol} \mathrm{~m}^{-3}$, according to eqn (3) (see Experimental section), the elastic modulus directly contributed by the covalent crosslinks is $1.5 \pm 0.6 \mathrm{MPa}$, much less than the measured modulus difference between the two groups. This suggests that direct contribution of covalent cross-links to $E_{\text {ind }}$ is not a dominant factor $(\approx 8 \%)$. Instead, the presence of permanent covalent cross-linking can alter the dynamics of ionic cross-links, providing synergistic effects to stiffen the network. Given the breakable nature of ionic cross-links, they undergo breaking and re-formation during the indentation. Here, $E_{\text {ind }}$ reflects an effective indentation resistance resulted from dynamics of ionic cross-links and viscosity of polymer chain segments. Therefore, the permanent anchorage endowed by covalent cross-links can provide additional resistance to polymer segment movement, and thus, reduce the extent of ionic cross-
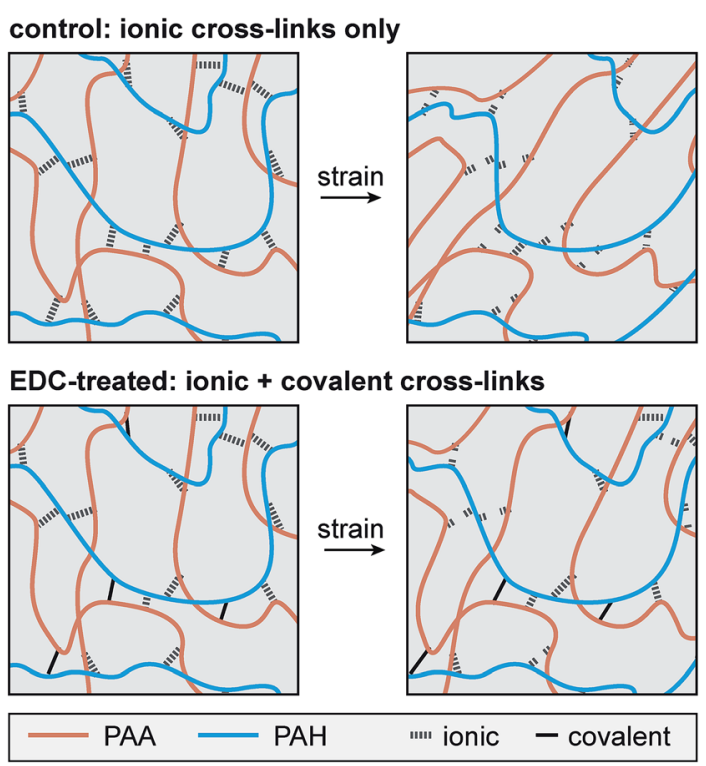

Fig. 7 Schematics illustrate the synergistic, non-additive impact of EDC-induced covalent cross-links on reducing the breaking and reformation of ionic cross-links in the PAH/PAA networks under mechanical stress and strain. link breaking, as illustrated in Fig. 7. This synergistic effect is likely to be the dominating mechanism leading to the increased $E_{\text {ind }}$, and the higher effective ionic cross-link density, $c_{\mathrm{ei}}$, for the EDC-treated network. Meanwhile, this effect is consistent across all net neutral states with both high (lower IS, $0.01 \mathrm{M}$ ) and low ionic cross-link densities (higher IS, $1.0 \mathrm{M})$ (Fig. 3b).

One key outcome from the relaxation test is the significant increase in the degree of elasticity, $E_{\infty} / E_{0}$, after covalent crosslinking (Fig. $4 \mathrm{a}$ and b). Similar to the case of $E_{\text {ind }}$, since the covalent cross-links do not directly contribute to $\approx 20 \%$ of the total cross-link density (e.g., only $\approx 1.6 \%$ at pH $5.5,0.01 \mathrm{M}$ ), this increased elasticity further highlights the synergistic, rather than additive, effect of covalent cross-linking (Fig. 7). During relaxation, the permanent anchorages can limit viscous flow of polymer segments and sustain additional molecular stresses on chain segments. This effect thus reduces ionic cross-link breaking and provides an amplified effect on the degree of elasticity. The synergistic impacts of covalent cross-linking are also illustrated by changes in viscoelastic relaxation time constants. For ionic networks, our previous study showed that the dominating, short term relaxation mode $\left(\tau_{1} \approx 50 \mathrm{~ms}\right)$, is governed by the breaking and re-formation of ionic bonds. ${ }^{30}$ With added covalent cross-links, the increase in $\tau_{1}$ (Fig. $5 \mathrm{c}$ ) is associated with the greater hindrance to polymer chain movement, as the permanent anchorage can decrease the mobility of polymer chain segments, and its associated ionic bond breaking or re-formation. This effect is also present for the less pronounced long-term relaxation $\left(\tau_{2}\right)$, as the permanent anchorage can also impact the kinetics of other viscoelastic mechanisms, such as reptation, disentanglement and fluid flow.

\subsection{Viscoelasticity at net charged states: differentiated impacts of covalent cross-linking}

The addition of covalent cross-links also shows pronounced, yet differentiated impacts on viscoelasticity at the highly swollen, net charged state, $\mathrm{pH} 2.0$, IS $0.01 \mathrm{M}$ (Table 1c). At this state, fixed charge-induced Donnan effect contributes to a higher degree of elasticity than the net neutral states for the control network $\left(E_{\infty} / E_{0}=40.2 \pm 2.1 \%\right)$. Here, adding covalent cross-links further elevates this ratio to $55.1 \pm 2.8 \%$, a similar additive impact as in net neutral states. On the other hand, contrary to the increased time constants at the net neutral states, the EDC-treated network shows no changes in $\tau_{1}$ and $\tau_{2}$ when compared to the control (Table 1c), illustrating differentiated impacts on viscoelasticity. This difference can be attributed to the different viscoelasticity mechanisms at the highly swollen state. At this state, given the loose ionic bonding and high degree of swelling, the time-dependent mechanics is dominated by polymer chain movement and fluid flow, rather than the breaking and re-formation of ionic cross-links. ${ }^{30,51}$ Therefore, since the control and EDC-treated networks are both highly swollen to a similar degree, the time constants associated with the chain segment movement 
and fluid flow are likely to be similar, leading to similar time constants.

\subsection{Viscoelasticity of polyelectrolyte networks with covalent cross-links}

At the most acidic state, $\mathrm{pH}=1.5$, all ionic cross-links break due to the full protonation of the carboxyl groups. The control network is fully dissolved, and the EDC-treated one becomes a classic type polyelectrolyte network, that is, a charged network held solely by covalently cross-links. ${ }^{52}$ Here, without the ionic cross-links, the network has the highest degree of elasticity ( $E_{\infty} / E_{0} \approx 70 \%$, Fig. $5 \mathrm{~b}$ and Table $1 \mathrm{~d}$ ), as viscoelasticity can only originate from polymer chain movement and fluid-polymer friction. At 1.0 M IS, the Donnan effect is minimized (Debye length $\kappa^{-1} \approx 0.3 \mathrm{~nm}$ ), and the equilibrium modulus, $E_{\infty}$, is determined by the network conformational entropy. Interestingly, even for this covalent network, rubber elasticity theory alone cannot predict the modulus change from IS $=1.0 \mathrm{M}$ to $0.01 \mathrm{M}$. Here, at $\mathrm{pH}=1.5$, despite the $1.31 \pm 0.06 \times$ change in $H$ suggests $\approx 24 \%$ decrease in $E_{\infty}$, value of $E_{\infty}$ instead increases for $\approx 17 \%$, highlighting the contribution of the Donnan osmotic pressure to the apparent modulus at the lower IS $\left(\kappa^{-1} \approx 3 \mathrm{~nm}\right)$. Meanwhile, at the two ionic strengths, the similarity of time constants suggests similar viscoelastic mechanisms. According to previous macroscale studies of covalent hydrogels, the dominating mechanism is likely to be the fluid flow-governed poroelasticity, especially for the short-term constant $\tau_{1} \cdot{ }^{53}$

Given the breakable nature of ionic cross-links, it is possible that the deformation mechanisms of ionic networks under creep can be distinctive from those under force relaxation. For example, constant-force creep can lead to not only viscoelastic, but also viscoplastic deformation, which has been shown for various biopolymer hydrogels at the macroscale. ${ }^{54}$ To this end, our ongoing studies are further probing the impact of covalent cross-linking on the viscoelastic and viscoplastic behaviors of ionic networks under creep. We anticipate these results will provide a more comprehensive understanding of the nanomechanics of polyelectrolyte networks held by various crosslink types.

\subsection{Limitation and outlook}

One limitation of this study is that the use of Hertz model does not account for mechanical nonlinearity of the polymer networks. A recent study shows that the neo-Hookean modelbased finite element analysis can better capture the nonlinearity of LbL polymer network elasticity. ${ }^{55}$ To minimize this nonlinearity, we limit our maximum indentation depth in the linear deformation regime (maximum indentation depth $D_{\max }<$ $0.1 H),{ }^{42}$ in which, the linear Hertz model is able to accurately account for the tip-sample contact geometry and fit the $F-D$ curve well $\left(R^{2}>0.98\right)$. Since our focus is on viscoelasticity, instead of material nonlinearity, we only study the linear regime by limiting maximum deformation $D_{\max }<0.4 R$. However, since polymer networks can have distinctive viscoelastic behaviors in the nonlinear regime, our ongoing studies aim to adapt more complex nonlinear polymer mechanics models ${ }^{56-58}$ to further probe the viscoelasticity in the non-linear regime.

Moreover, we were not able to directly quantify the portion of carboxyl-amine ionic pairs that are converted to the covalent amide bonds. Since the peak wavenumbers for amide bonds $\left(\sim 1700-1600 \text { and } 1580-1510 \mathrm{~cm}^{-1}\right)^{48}$ are overlapping with those for carboxyl bonds ( 1710-1700 and 1565$\left.1542 \mathrm{~cm}^{-1}\right)^{33}$ and amine bonds $(\sim 1629-1626$ and 1610$\left.1586 \mathrm{~cm}^{-1}\right),{ }^{59}$ we cannot distinguish the two in the fashion like we delineate the protonated and charged carboxyl groups (in Fig. 2). In addition, even if the molar ratio of amides and un-converted carboxyl groups can be deduced from FTIR, this ratio does not directly represent the ratios between covalent and ionic bonds that contribute to the polyelectrolyte network modulus. This is because, first, the carboxyl groups that contribute to ionic cross-links are highly dependent on the degree of ionization ${ }^{33}$ and "doping" effect by "extrinsic" ion pairing with free ions. ${ }^{34}$ Second, ionic and covalent bonds that do not contribute to the network formation, e.g., the "ladderforming" bonds, ${ }^{46}$ do not contribute to the mechanical properties of the network, while the "network-forming" and "ladder-forming" bonds cannot be distinguished from the FTIR results. These challenges prevented us from directly quantifying the densities of ionic and covalent cross-links at each pH and IS state at the molecular level. However, this limitation was addressed by comparison of the "effective cross-link density" deduced from the elastic modulus (Fig. 6). According to these results, it is clear that the density of covalent cross-links is very low in comparison to the ionic bonds (Fig. 6a). Despite this, we still detected substantial changes in the viscoelastic behaviors (Fig. 4 and 5), highlighting the non-additive, synergistic effects of the two crosslink types.

\section{Conclusions}

This study revealed the impacts of adding covalent cross-linking on the nanomechanical viscoelasticity of ionically cross-linked polyelectrolyte networks. Through modulating solution $\mathrm{pH}$ and IS, we probed the different effects of covalent cross-links on ionic networks at various cross-link and fixed charge densities. The addition of covalent cross-linking, despite at relatively low concentration, can substantially increase both the elastic modulus and the degree of elasticity in a synergistic, nonadditive manner. At less swollen, net neutral states, adding covalent cross-linking increases relaxation time constants through providing greater hindrance to polymer chain reorganization and reducing ionic cross-link breaking/re-formation. At the highly swollen, net charged state, this modification does not substantially alter the time constants, possibly due to similar viscoelastic mechanisms with or without covalent crosslinking. These results highlighted the unique viscoelastic nanomechanics of polyelectrolyte networks with hybrid crosslink types. Since mechanical characteristics are key determinants of many stimulus-responsive biomaterial-based applications, ${ }^{\mathbf{6 0}, 61}$ this study provides a basic knowledge foundation for 
a better informed design of polymer hydrogels and LbL films at the microscale.

\section{Conflicts of interest}

There are no conflicts to declare.

\section{Acknowledgements}

This work was supported by the Faculty Start-up Grant at Drexel University (LH), the Faculty Start-up Grant at Temple University (JY), and NSF DMR-1055594 (DL). It was also supported by the Drexel Areas of Research Excellence (DARE) initiative (LH). We thank the Nano/Bio Interface Center at the University of Pennsylvania for the use of MFP-3D.

\section{References}

1 L. Richert, P. Lavalle, D. Vautier, B. Senger, J. F. Stoltz, P. Schaaf, J. C. Voegel and C. Picart, Biomacromolecules, 2002, 3, 1170-1178.

2 J. D. Mendelsohn, S. Y. Yang, J. Hiller, A. I. Hochbaum and M. F. Rubner, Biomacromolecules, 2003, 4, 96-106.

3 A. J. Engler, L. Richert, J. Y. Wong, C. Picart and D. E. Discher, Surf. Sci., 2004, 570, 142-154.

4 D. E. Discher, D. J. Mooney and P. W. Zandstra, Science, 2009, 324, 1673-1677.

5 K. F. Ren, T. Crouzier, C. Roy and C. Picart, Adv. Funct. Mater., 2008, 18, 1378-1389.

6 P. Schultz, D. Vautier, L. Richert, N. Jessel, Y. Haikel, P. Schaaf, J. C. Voegel, J. Ogier and C. Debry, Biomaterials, 2005, 26, 2621-2630.

7 J. Zhang and D. M. Lynn, Macromolecules, 2006, 39, 89288935.

8 J. Hiller, J. D. Mendelsohn and M. F. Rubner, Nat. Mater., 2002, 1, 59-63.

9 X. Zhu, D. Janczewski, S. S. Lee, S. L. Teo and G. J. Vancso, ACS Appl. Mater. Interfaces, 2013, 5, 5961-5968.

10 X. Y. Cao, M. E. Pettitt, F. Wode, M. P. A. Sancet, J. H. Fu, J. A. Ji, M. E. Callow, J. A. Callow, A. Rosenhahn and M. Grunze, Adv. Funct. Mater., 2010, 20, 1984-1993.

11 K. C. Wood, J. Q. Boedicker, D. M. Lynn and P. T. Hammond, Langmuir, 2005, 21, 1603-1609.

12 C. J. Detzel, A. L. Larkin and P. Rajagopalan, Tissue Eng., Part $B, 2011,17,101-113$.

13 S. Jana, B. J. Tefft, D. B. Spoon and R. D. Simari, Acta Biomater., 2014, 10, 2877-2893.

14 G. Decher, Science, 1997, 277, 1232-1237.

15 W. B. Stockton and M. F. Rubner, Macromolecules, 1997, 30, 2717-2725.

16 N. A. Kotov, Nanostruct. Mater., 1999, 12, 789-796.

17 T. Boudou, T. Crouzier, K. F. Ren, G. Blin and C. Picart, Adv. Mater., 2010, 22, 441-467.

18 J. J. Harris, P. M. DeRose and M. L. Bruening, J. Am. Chem. Soc., 1999, 121, 1978-1979.
19 T. L. Sun, T. Kurokawa, S. Kuroda, A. B. Ihsan, T. Akasaki, K. Sato, M. A. Haque, T. Nakajima and J. P. Gong, Nat. Mater., 2013, 12, 932-937.

20 Y. Chen and K. Shull, Macromolecules, 2017, 50, 3637-3646.

21 H. Peng, K. Rubsam, F. Jakob, U. Schwaneberg and A. Pich, Biomacromolecules, 2016, 17, 3619-3631.

22 W. H. Kuo, M. J. Wang, H. W. Chien, T. C. Wei, C. Lee and W. B. Tsai, Biomacromolecules, 2011, 12, 4348-4356.

23 M. D. Moussallem, S. G. Olenych, S. L. Scott, T. C. S. Keller III and J. B. Schlenoff, Biomacromolecules, 2009, 10, 3062-3068.

24 L. Richert, A. J. Engler, D. E. Discher and C. Picart, Biomacromolecules, 2004, 5, 1908-1916.

25 A. Schneider, G. Francius, R. Obeid, P. Schwinte, J. Hemmerle, B. Frisch, P. Schaaf, J. C. Voegel, B. Senger and C. Picart, Langmuir, 2006, 22, 1193-1200.

26 Y. Min and P. T. Hammond, Chem. Mater., 2011, 23, 53495357.

27 D. E. Discher, P. Janmey and Y. L. Wang, Science, 2005, 310, 1139-1143.

28 D. Mertz, J. Hemmerle, J. Mutterer, S. Ollivier, J. C. Voegel, P. Schaaf and P. Lavalle, Nano Lett., 2007, 7, 657-662.

29 L. Xiao, J. Zhu, D. J. Londono, D. J. Pochan and X. Jia, Soft Matter, 2012, 8, 10233-10237.

30 B. Han, D. R. Chery, J. Yin, X. L. Lu, D. Lee and L. Han, Soft Matter, 2016, 12, 1158-1169.

31 S. S. Shiratori and M. F. Rubner, Macromolecules, 2000, 33, 4213-4219.

32 S. E. Burke and C. J. Barrett, Langmuir, 2003, 19, 3297-3303.

33 J. Choi and M. F. Rubner, Macromolecules, 2005, 38, 116-124.

34 T. R. Farhat and J. B. Schlenoff, J. Am. Chem. Soc., 2003, 125, 4627-4636.

35 P. C. Hiemenz and R. Rajagopalan, in Principles of Colloid and Surface Chemistry, Marcel Dekker, New York, NY, 1997, ch. 11, pp. 499-533.

36 D. G. Hoare and D. E. Koshland Jr, J. Biol. Chem., 1967, 242, 2447-2453.

37 T. L. Smith, J. Polym. Sci., Polym. Symp., 1974, 46, 97-114.

38 P. V. Pavoor, A. Bellare, A. Strom, D. Yang and R. E. Cohen, Macromolecules, 2004, 37, 4865-4871.

39 P. Schuetz and F. Caruso, Adv. Funct. Mater., 2003, 13, 929937.

40 S. A. Sukhishvili and S. Granick, Macromolecules, 2002, 35, 301-310.

41 R. W. Farndale, D. J. Buttle and A. J. Barrett, Biochim. Biophys. Acta, 1986, 883, 173-177.

42 E. K. Dimitriadis, F. Horkay, J. Maresca, B. Kachar and R. S. Chadwick, Biophys. J., 2002, 82, 2798-2810.

43 M. Ahearne, Y. Yang, A. J. El Haj, K. Y. Then and K. K. Liu, J. R. Soc., Interface, 2005, 2, 455-463.

44 C. Zener, Elasticity and Anelasticity of Metals, Chicago University Press, Chicago, IL, 1948.

45 P. J. Flory, in Principles of Polymer Chemistry, Cornell University Press, 1953, pp. 432-494.

46 J. A. Jaber and J. B. Schlenoff, J. Am. Chem. Soc., 2006, 128, 2940-2947.

47 J. A. Jaber and J. B. Schlenoff, Macromolecules, 2005, 38, 1300-1306. 
48 J. L. R. Arrondo, A. Muga, J. Castresana and F. M. Goñi, Prog. Biophys. Mol. Biol., 1993, 59, 23-56.

49 C. Wu, S. Aslan, A. Gand, J. S. Wolenski, E. Pauthe and P. R. Van Tassel, Adv. Funct. Mater., 2013, 23, 66-74.

50 R. M. Silverstein, F. X. Webster and D. J. Kiemle, in Spectrometric Identification of Organic Compounds, John Wiley \& Sons, Hoboken, NJ, 7th edn, 2005, pp. 72-126.

51 J. A. Jaber and J. B. Schlenoff, Chem. Mater., 2006, 18, 57685773.

52 P. J. Flory, in Principles of Polymer Physical Chemistry, Cornell University Press, 1953, pp. 595-639.

53 Y. Lai and Y. Hu, Soft Matter, 2017, 13, 852-861.

54 S. Nam, J. Lee, D. G. Brownfield and O. Chaudhuri, Biophys. J., 2016, 111, 2296-2308.
55 T. Sherstova, B. T. Stokke, B. Skallerud, G. Maurstad and V. E. Prot, Soft Matter, 2016, 12, 7338-7349.

56 Y. Takano, Polym. J., 1974, 6, 61-71.

57 S. Hayashi, Polym. J., 1985, 17, 597-606.

58 E. M. Arruda and M. C. Boyce, J. Mech. Phys. Solids, 1993, 41, 389-412.

59 A. Barth, Prog. Biophys. Mol. Biol., 2000, 74, 141-173.

60 M. A. Cohen Stuart, W. T. S. Huck, J. Genzer, M. Müller, C. Ober, M. Stamm, G. B. Sukhorukov, I. Szleifer, V. V. Tsukruk, M. Urban, F. Winnik, S. Zauscher, I. Luzinov and S. Minko, Nat. Mater., 2010, 9, 101-113.

61 M. Wang, Y. Gao, X. Li and M. J. Serpe, Polym. Chem., 2017, 8, 127-143. 